\title{
Mathematical modelling of Stefan Phase Change for Thermal Dissociation of Natural Gas Hydrate
}

\author{
MingChuan $\operatorname{Li}^{1}$ \\ ${ }^{1}$ China University of Petroleum East China - Qingdao Campus
}

October 22,2020

\begin{abstract}
The thermal dissociation process of natural gas hydrate is a Stefan phase change problem with moving boundaries. Based on the heat conduction model in integral form, for the continuous single-phase hydrate control volume, an energy conservation condition involved interfacial coupling Stefan is built, for the control volume of the thermal dissociation natural gas hydrate with a sharp moving boundary. A Neumann solution of Stefan phase change model has been obtain, for the thermal dissociation in a semi-infinite natural gas hydrate reservoir, using Boltzmann similar variables; and the uniqueness is determined for the Stefan model, by monotonic proving of the transcendental equation. By example analysis, the monotonicity of the transcendental equation and the uniqueness solution of Stefan model, have been verified. By MATLAB programmes, the laws of temperature distribution, dissociation frontal brim, and the sensitivity parameter fitting of temperature have been studied during the thermal dissociation process of a hydrate reservoir.
\end{abstract}

\section{Hosted file}

4.Manuscript(initial).pdf available at https://authorea.com/users/369261/articles/488187mathematical-modelling-of-stefan-phase-change-for-thermal-dissociation-of-natural-gashydrate 